\title{
Near-infrared fluorescent image-guided surgery for intracranial meningioma
}

\author{
John Y. K. Lee, MD, MSCE,,4 John T. Pierce, MS, Jayesh P. Thawani, MD, ${ }^{1}$ Ryan Zeh, BS, ${ }^{1}$ \\ Shuming Nie, PhD, ${ }^{5}$ Maria Martinez-Lage, MD, ${ }^{3}$ and Sunil Singhal, MD ${ }^{2,4}$
}

Departments of ${ }^{1}$ Neurosurgery, ${ }^{2}$ Surgery, and ${ }^{3}$ Pathology, Hospital of the University of Pennsylvania; ${ }^{4}$ Center for Precision Surgery, Abramson Cancer Center, University of Pennsylvania School of Medicine, Philadelphia, Pennsylvania; and ${ }^{5}$ Department of Chemistry, Emory University School of Medicine, Atlanta, Georgia

\begin{abstract}
OBJECTIVE Meningiomas are the most common primary tumor of the central nervous system. Complete resection can be curative, but intraoperative identification of dural tails and tumor remnants poses a clinical challenge. Given data from preclinical studies and previous clinical trials, the authors propose a novel method of localizing tumor tissue and identifying residual disease at the margins via preoperative systemic injection of a near-infrared (NIR) fluorescent contrast dye. This technique, what the authors call "second-window indocyanine green" (ICG), relies on the visualization of ICG approximately 24 hours after intravenous injection.
\end{abstract}

METHODS Eighteen patients were prospectively identified and received $5 \mathrm{mg} / \mathrm{kg}$ of second-window ICG the day prior to surgery. An NIR camera was used to localize the tumor prior to resection and to inspect the margins following standard resection. The signal to background ratio (SBR) of the tumor to the normal brain parenchyma was measured in triplicate. Gross tumor and margin specimens were qualitatively reported with respect to fluorescence. Neuropathological diagnosis served as the reference gold standard to calculate the sensitivity and specificity of the imaging technique.

RESULTS Eighteen patients harbored 15 WHO Grade I and 3 WHO Grade II meningiomas. Near-infrared visualization during surgery ranged from 18 to 28 hours (mean 23 hours) following second-window ICG infusion. Fourteen of the 18 tumors demonstrated a markedly elevated SBR of $5.6 \pm 1.7$ as compared with adjacent brain parenchyma. Four of the 18 patients showed an inverse pattern of NIR signal, that is, stronger in the adjacent normal brain than in the tumor (SBR $0.31 \pm 0.1$ ). The best predictor of inversion was time from injection, as the patients who were imaged earlier were more likely to demonstrate an appropriate SBR. The second-window ICG technique demonstrated a sensitivity of $96.4 \%$, specificity of $38.9 \%$, positive predictive value of $71.1 \%$, and a negative predictive value of $87.5 \%$ for tumor.

CONCLUSIONS Systemic injection of NIR second-window ICG the day before surgery can be used to visualize meningiomas intraoperatively. Intraoperative NIR imaging provides higher sensitivity in identifying meningiomas than the unassisted eye. In this study, 14 of the 18 patients with meningioma demonstrated a strong SBR compared with adjacent brain. In the future, reducing the time interval from dye injection to intraoperative imaging may improve fluorescence at the margins, though this approach requires further investigation.

Clinical trial registration no.: NCT02280954 (clincialtrials.gov).

https://thejns.org/doi/abs/10.3171/2016.10.JNS161636

KEY WORDS fluorescence; brain tumor; meningioma; indocyanine green; ${ }^{125} \mathrm{R}$; near infrared; oncology

$\mathrm{I}$ N the United States, approximately 15,000 patients undergo brain tumor resection each year. ${ }^{2}$ Meningiomas are the most frequently diagnosed primary brain tumor, accounting for $33.8 \%$ of all primary central nervous system tumors in the United States. ${ }^{7}$ Most are encapsulated and benign (WHO Grade I), but even these tumors can still cause serious morbidity based on their location and compression of adjacent neurovascular structures. ${ }^{7,27}$ In addition, atypical (WHO Grade II) and malignant (WHO Grade III) meningiomas account for approximately 5\%-20\% of all diagnosed meningiomas, depending on the grading scheme., ${ }^{4,7}$ The gold-standard treatment for symptomatic meningioma

ABBREVIATIONS 5-ALA = 5-aminolevulinic acid; EPR = enhanced permeability and retention; ICG = indocyanine green; NIR = near-infrared dye; NPV = negative predictive value; $P P V=$ positive predictive value; $R O C=$ receiver operating characteristic; $S B R=$ signal to background ratio.

SUBMITTED June 21, 2016. ACCEPTED October 4, 2016.

INCLUDE WHEN CITING Published online April 7, 2017; DOI: 10.3171/2016.10.JNS161636. 
is resection, ${ }^{27,31}$ but even after surgical removal and maximal adjunctive radiation therapy, the median time to radiological progression is 12,7 , and 2 years for WHO Grade I, II, and III meningiomas, respectively. ${ }^{29}$ Although differentiating tumor tissue from surrounding normal brain tissue is not generally considered a major challenge during resection, the ability to distinguish dural tails, optic canal invasion, and brain invasion could benefit from improvements in preoperative tumor identification.

Several surgical adjuncts that assist with meningioma resection include neuronavigational systems correlated with preoperative radiological images ${ }^{30}$ and fluorescenceguided imaging with 5-aminolevulinic acid (5-ALA). ${ }^{35,37,40}$ A prodrug in the porphyrin family, 5-ALA has been shown to facilitate tumor identification, improve grosstotal resection rates, and prolong progression-free survival outcomes in meningiomas ${ }^{8}$ and glioblastomas.$^{36}$ However, 5-ALA has limitations because it fluoresces in the visible light spectrum, lacks tissue penetrance, is confounded by background brain autofluorescence, and shows poor optical contrast since visualization of normal tissue with blue light only is limited ${ }^{35,38,40}$

Indocyanine green (ICG) has been used as a vascular contrast agent in the assessment of meningioma perfusion and venous drainage, but it has not been successfully used as a contrast agent for the meningioma itself. Its role has been primarily as a dye for videoangiography, ${ }^{9,11,19}$ in which a bolus dose of ICG is intravenously delivered minutes before visualization. Conversely, we have studied an alternative approach that relies on "second-window ICG," in which a higher dose of ICG $(5 \mathrm{mg} / \mathrm{kg}$ [350 $\mathrm{mg}$ for a 70$\mathrm{kg}$ adult] as compared with the usual 25-mg intravenous angiography bolus) is injected 1 day prior to surgery. This technique reveals unique properties of the near-infrared (NIR) dye. To differentiate the vascular properties of ICG from the second window of ICG, we call the technique "second-window ICG." Over 24 hours, the dye accumulates in tumor tissue because of an enhanced permeability and retention (EPR) effect.111 Given data from preclinical animal studies and clinical trials, we optimized ICG dosing and timing for the visualization of tumors using this second-window technique. In a rodent flank tumor model, Jiang et al. demonstrated that tumor could not be visualized at the usual videoangiography doses; however, optimal tumor contrast was achieved at 24 hours after the injection of $5-10 \mathrm{mg} / \mathrm{kg}$ of ICG. ${ }^{18}$

Using data from this rodent study, as well as data from patients with lung cancer, we administered second-window ICG in patients with intracranial meningiomas. ${ }^{12,22,24}$ In the present study, we hypothesized that second-window ICG would localize meningiomas in situ against the normal brain background and could be used to identify margins intraoperatively. ${ }^{13-15}$ We report on the first 18 cases involving NIR second-window ICG as a tumor contrast agent for meningiomas in humans and as a novel imaging strategy that utilizes the EPR effect. ${ }^{12,22,24}$

\section{Methods}

\section{Study Design}

This prospective cohort study was approved by the Uni- versity of Pennsylvania Institutional Review Board, and all patients gave informed consent. The trial is registered with the ClinicalTrials.gov database (http://clinicaltrials. gov), and its registration no. is NCT02280954. Recruitment started in October 2014. Any adult (age $>18$ years) with an imaging diagnosis of meningioma and undergoing craniotomy was considered eligible for this study. Pregnancy and iodide allergy were the main exclusion criteria. All patients underwent preoperative MRI of the brain with intravenous gadolinium. The presence or absence of gadolinium enhancement and the tumor size on MRI were noted. Patients were informed that the scope of surgery would not substantially change based on the NIR findings and that biopsies would be taken only if deemed safe by the senior surgeon (J.Y.K.L.).

\section{NIR Contrast Agent}

Patients were injected intravenously with a $5-\mathrm{mg} / \mathrm{kg}$ dose of ICG (C43H47N2O6S2.Na, Akorn Pharmaceuticals) between 18 and 28 hours (mean 23 hours) prior to surgery. This time interval was selected based on preclinical studies from our laboratory. ${ }^{21,34}$ There were no toxicities from the second-window ICG administered at this elevated dose.

\section{NIR Imaging System}

All cases were imaged using the VisionSense iridium camera system, which is FDA-approved for perfusion imaging in plastic and reconstructive surgery (to assess for flap viability, for example) and in this study was used off-label for NIR imaging of the brain after institutional review board approval had been obtained. The excitation source was a laser tuned in the NIR range $(805 \mathrm{~nm})$. The sensor was a silicon image sensor with an open field of view of $19 \times 14 \mathrm{~cm}$ at a $40-\mathrm{cm}$ nominal imaging distance. The emission filter band in the visible light range was from 400 to $700 \mathrm{~nm}$, whereas the emission filter band for NIR was much narrower from 825 to $850 \mathrm{~nm}$. A heat map was used as an overlay on the visible-light image to provide quantitative fluorescence intensity. Image processing was performed in real time and displayed at $1080 \mathrm{p}$ video resolution (with recording at 720p). In 15 cases a standard VisionSense open camera system was used, and in 3 cases an endoscope with a 4-mm outer diameter was used.

The endoscope featured a dual optical path design in which the white light and NIR optical paths were completely separate and independent. White light and NIR images were transferred through their respective paths from the surgical field through the tip of the endoscope to the NIR fluorescence camera. The NIR fluorescence camera was a dual path device with separate paths for the visible light and NIR images. The net effect of this design was the complete isolation of the NIR fluorescence signals from the white light signals, allowing very faint fluorescence images to be acquired with high quality in the presence of very strong white light.

\section{Study Procedure}

Patients were placed under anesthesia and underwent craniotomy utilizing anatomical landmarks as well as 
neuronavigational imaging. Preoperative MRI was used for navigation in all subjects. Once the bone flap was removed and the dura mater exposed, the operating room lights were dimmed. This is an important step because standard operating room lights-whether halogen, xenon, or tungsten-all produce light with wavelengths in the NIR spectrum. Similarly, the standard optical tracking systems used for neuronavigation utilize the NIR wavelength to triangulate position, and thus the optical emitters/trackers were pointed to the floor, away from the operative field. All window shades were closed to limit natural light in the room. The NIR imaging system was sterilely draped and positioned above the operative field. The presence or absence of NIR signal was recorded. The dura was then opened under visible light, and the tumor was visualized. Room lights were again shut off, and the NIR camera was brought into the operative field, and the ability to localize the NIR signal corresponding to the tumor prior to cortical incision was qualitatively recorded in a yes/no format. Surgery then proceeded in the standard manner without the use of NIR imaging adjuncts.

After the tumor was resected and the attending neurosurgeon was satisfied that complete resection had been achieved, NIR imaging was used to identify areas of residual disease. The surgeon's impression of the NIR signal of the tumor itself and the margin biopsy samples was qualitatively measured (yes/no) and photographically documented for post hoc analysis. Areas were biopsied at the discretion of the senior surgeon (J.Y.K.L.).

While still in the operating room, the attending surgeon (J.Y.K.L.) coded all specimens as consistent with tumor under bright light (white: yes/no) and under NIR fluorescence (NIR: yes/no). The histopathological diagnosis obtained several days later served as the gold standard and was cataloged by a single neuropathologist (M.M.L.) at our institution. The presence of tumor was noted and incorporated into our analysis.

Patients were admitted to the intensive care unit following surgery. There were no adverse outcomes. Postoperative MRI took place on the 1st postoperative day. Patients were seen approximately 2 and 4 weeks after surgery.

\section{Immunohistochemistry and Fluorescence Microscopy}

Frozen tumor sections were prepared in a standard fashion. Samples were examined using an Olympus IX51 fluorescence microscope equipped with an ICG-specific filter set (Chroma 49030). Histopathological analyses were performed, and tissue samples that showed fluorescence following the intended resection were catalogued. Permanent sections were chemically fixed and embedded in paraffin blocks at a 5- $\mu \mathrm{m}$ thickness. Vertical sectioning was performed along with $\mathrm{H} \& \mathrm{E}$ staining. Each tissue sample was assessed for the presence of tumor-specific cells (matched to the bulk sample).

\section{Data Analysis}

The data were analyzed using Stata statistical software version 10 (StataCorp LLC). To quantitate the amount of fluorescence from the tissue, we used region of interest analyses within the VisionSense software (VSPlayer v1.8.05.01). A background reading was taken from adjacent normal dura or brain tissue to generate a signal to background ratio (SBR).

Two-by-two contingency tables were constructed using Stata version 10, and sensitivity, specificity, positive predictive value (PPV), negative predictive value (NPV), and receiver operating characteristic (ROC) analysis were computed. By calculating the area under the curve generated by plotting true-positive (y) versus false-positive (x) rates, we were able to determine the test discrimination, which reflects the ability of the test to properly classify those without and those with meningioma. ${ }^{20}$

\section{Results \\ Clinical Data}

Eighteen patients were enrolled in this study. Thirteen patients were female, and the mean age overall was 55 years old (range 20-74 years). Eleven patients had convexity meningiomas, and 1 patient had a parasagittal frontal meningioma. The remaining 6 patients had skull base or intraventricular meningiomas: 2 had tuberculum sella meningiomas, 1 had an olfactory groove meningioma, 1 had a cerebellopontine angle meningioma, 1 had an intraventricular meningioma, and 1 had a clinoid (medial sphenoid wing) meningioma (Table 1). All patients tolerated injection of the second-window ICG at $5 \mathrm{mg} / \mathrm{kg}$, administered 1 day prior to surgery without adverse event. On the final pathological analysis, 15 patients had WHO Grade I meningioma and 3 had WHO Grade II meningioma. The majority of tumors (78\%) were meningothelial meningiomas, but there were also 3 transitional type and 1 psammomatous type.

\section{Intraoperative NIR Fluorescence: SBR}

Using the second-window ICG technique, we first sought to determine whether any NIR fluorescence could be detected within the meningioma at all. Typically, ICG in NIR imaging is intended for use within minutes of its injection. We found that despite administering secondwindow ICG 1 day prior to surgery, we were able to visualize a strong NIR signal in $14(78 \%)$ of the 18 patients. In these 14 patients, there was a significantly stronger NIR signal within the tumor than in the surrounding brain parenchyma. The mean SBR in these 14 patients was $5.6 \pm$ 1.7. Moreover, the NIR signal in these patients could be visualized prior to opening the dura, although the mean SBR before opening the dura was $4.6 \pm 1.9$. As seen in Fig. 1, localization of the NIR signal corresponded to the tumor location, and after the dura was opened, the SBR increased and was clearly confined to the meningioma location as opposed to the adjacent brain parenchyma or the reflected dura. Using a standard neurosurgical technique with visible light, we removed meningioma from the brain and imaged it on the back table. The tumor continued to demonstrate strong NIR fluorescence (Fig. 2F).

\section{Intraoperative NIR Fluorescence: Inverse SBR}

The majority of patients with meningiomas (78\%) demonstrated a strong NIR signal that appeared to be confined to the tumor and appeared to have a strong SBR. However, 
TABLE 1. Summary of patient, tumor, and imaging characteristics

\begin{tabular}{|c|c|c|c|c|c|c|c|c|c|}
\hline Case No. & $\begin{array}{l}\text { Age } \\
\text { (yrs) }\end{array}$ & Sex & $\begin{array}{l}\text { Weight } \\
(\mathrm{kg})\end{array}$ & BMI & $\begin{array}{c}\text { Prior } \\
\text { Craniotomy } \\
\text { (yrs ago) }\end{array}$ & $\begin{array}{c}\text { Prior } \\
\text { Radiation } \\
\text { (yrs ago) }\end{array}$ & $\begin{array}{c}\text { Final Pathological } \\
\text { Type }\end{array}$ & Location & $\begin{array}{l}\text { ICG Dose } \\
\text { Injected } \\
\text { (mg) }\end{array}$ \\
\hline \multicolumn{10}{|c|}{ Meningioma WHO Grade I } \\
\hline $1 S$ & 40 & $\mathrm{~F}$ & 63.5 & 23.9 & & & Meningothelial & Convexity & 317 \\
\hline $13 S$ & 46 & $\mathrm{~F}$ & 75.8 & 34.0 & & & Meningothelial & Convexity & 377 \\
\hline 9 & 50 & $\mathrm{~F}$ & 69.4 & 27.8 & & & Meningothelial & Convexity & 347 \\
\hline 21 & 74 & M & 91.5 & 28.0 & & & Transitional & Convexity & 458 \\
\hline 22 & 57 & $\mathrm{~F}$ & 78.5 & 28.7 & & & Meningothelial & Convexity & 393 \\
\hline 24 & 53 & M & 108.0 & 34.3 & & & Transitional & Convexity & 541 \\
\hline 29 & 73 & $\mathrm{~F}$ & 49.4 & 19.0 & & & Meningothelial & Convexity & 247 \\
\hline 31 & 38 & $\mathrm{~F}$ & 79.8 & 29.1 & & & Meningothelial & Convexity & 399 \\
\hline 37 & 66 & $\mathrm{~F}$ & 68.0 & 27.4 & 0.5 & 22 & Meningothelial & Convexity & 340 \\
\hline 40 & 64 & $\mathrm{~F}$ & 60.8 & 24.5 & & & Transitional & Convexity & 304 \\
\hline 1 & 32 & $\mathrm{~F}$ & 65.3 & 24.2 & & & Meningothelial & Olfactory groove & 328 \\
\hline 32 & 57 & $\mathrm{~F}$ & 90.7 & 33.7 & & & Psammomatous & Tuberculum sella & 454 \\
\hline 10 & 69 & $\mathrm{~F}$ & 97.5 & 36.6 & & & Meningothelial & Cerebellar pontine angle & 488 \\
\hline 12 & 20 & M & 38.6 & 22.0 & & 19 & Meningothelial & $\begin{array}{l}\text { Intraventricular (radiation } \\
\text { induced) }\end{array}$ & 193 \\
\hline 33 & 62 & $\mathrm{~F}$ & 52.2 & 24.7 & & & Meningothelial & Clinoid (sphenoid wing) & 261 \\
\hline \multicolumn{10}{|c|}{ Meningioma WHO Grade II } \\
\hline $6 S$ & 70 & M & 91.2 & 27.2 & & & Meningothelial & Convexity & 456 \\
\hline 17 & 69 & M & 104.3 & 28.3 & 2 & 2.5 & Meningothelial & Tuberculum sella & 518 \\
\hline 3 & 54 & $\mathrm{~F}$ & 64.4 & 23.0 & 4 & 6.75 & Meningothelial & Parasagittal & 323 \\
\hline
\end{tabular}

a minority (4 patients [22\%]) demonstrated an inverse pattern of NIR signal (Fig. 2). In these 4 cases, the immediate adjacent brain parenchyma demonstrated higher NIR signal than the tumor itself (Cases 9, 22, 29, and 33 in Tables 1 and 2). The mean SBR in these inverted cases was less than $1(0.31 \pm 0.1)$, with the brain parenchyma background intensity (denominator) demonstrating greater NIR signal than the tumor signal (numerator). To understand the inversion pattern of NIR dye accumulation, we considered several explanations.

We first noted that this pattern of inversion might be related to sex, WHO grade, or a history of prior surgery or radiation. Specifically, none of the male patients (5 patients) with meningioma demonstrated an inversion pattern. None of the atypical WHO Grade II meningiomas (3 cases) demonstrated an inversion pattern, and no patient with a history of prior surgery or radiation (4 cases) demonstrated such a pattern. Thus, all the inversions occurred in benign (WHO Grade I) meningiomas in women who had not had prior surgery or radiation. In addition to these factors, we considered other variables that could serve as predictors of this inversion pattern.

Using logistic regression as a technique to predict an inversion event, we concluded that several variables did not predict inversion and found that one specific variable did predict it. Specifically, maximum tumor diameter (mm), patient age (years), presence of peritumoral T2/FLAIR signal on preoperative MRI (yes/no), T1 signal intensity ratio as compared with contralateral normal brain, location of meningioma (convexity vs other), body mass index (continuous variable), Ki-67 (continuous variable), and meningioma pathological subtype (WHO Grade I vs Grade II) did not predict inversion on logistic regression (Stata version 10). The only variable that appeared to approach but did not reach statistical significance $(p<0.25)$ was the time (hours) from ICG infusion to visualization (OR 1.34, $\mathrm{p}=0.1921$, univariate logistic regression). All patients were injected the day before surgery, but the actual number of hours from the infusion of second-window ICG to tumor visualization varied from 18 to 28.3 hours. This variability was related to scheduling and the imperfect prediction of when a patient might undergo surgery. Interestingly, none of the 4 patients imaged before 21 hours demonstrated inversion. In contrast, 4 (28.5\%) of the 14 patients imaged 21 hours after injection of the NIR contrast dye demonstrated inversion, suggesting that the dye had accumulated in the meningioma but then diffused out. The mean number of hours from injection to imaging for patients with the expected pattern of NIR fluorescence was 22.4 hours versus 24.4 hours in the patients with inversion $(\mathrm{p}=0.2256$, $\mathrm{t}$-test).

We studied time as a predictor of the inversion event using linear regression as well (Fig. 3). A univariate linear regression plot of SBR versus time from injection was predictive ( $p=0.022$, but $\left.\mathrm{R}^{2}=0.2876\right)$. In addition, a forward stepwise linear regression model incorporating the multiple variables tested in the prior logistic regression model yielded 2 significant variables for predicting SBR: sex and time to infusion ( $\mathrm{p}$ for infusion time $=0.056$, coefficient $=$ $-0.436)$. Hence, time from injection is a predictor of SBR as well as inversion. 

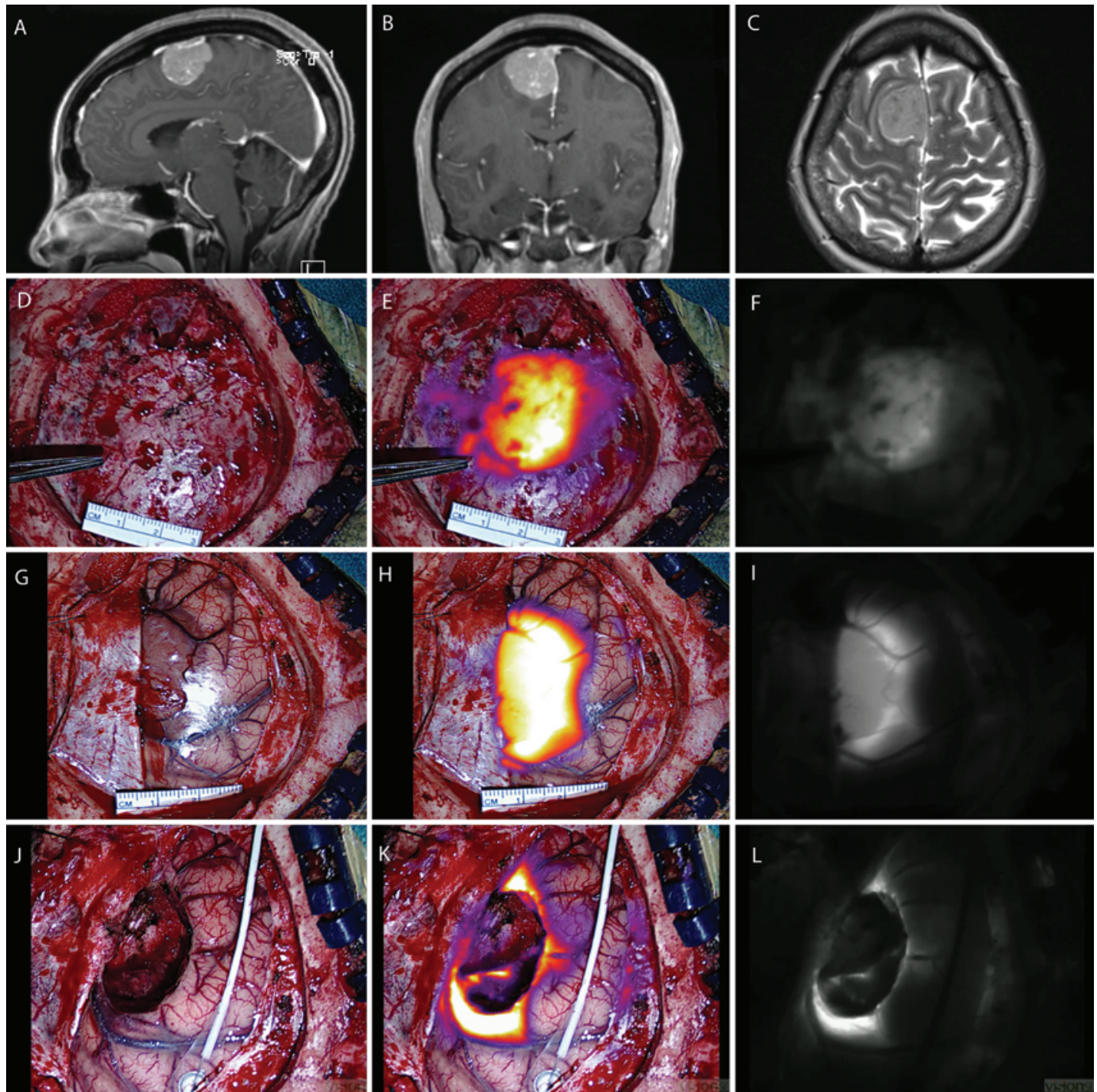

FIG. 1. Case 1S. Convexity meningioma displaying NIR fluorescence. Sagittal (A) and coronal (B) Gd-enhanced T1-weighted MR images. Axial T2-weighted MR image (C) demonstrating minimal adjacent edema. Visible-light image (D), NIR fluorescence superimposed and color-mapped onto the visible-light view $(\mathbf{E})$, and NIR image $(\mathbf{F})$ showing closure of the dura, although some NIR signal can be seen through the dura and localized to tumor. Visible-light image (G), NIR fluorescence superimposed and color-mapped onto the visible-light view $(\mathbf{H})$, and NIR image (I) showing dura reflected away and the tumor-brain interface. The SBR of NIR tumor signal is 6.05. Visible-light image $(\mathrm{J})$, NIR fluorescence superimposed and color-mapped onto the visible-light view $(\mathbf{K})$, and NIR image (L) showing residual fluorescence in anterior and posterior margins after resection of the main tumor. No fluorescence is seen in the midline falx. Figure is available in color online only.

Although the tumors demonstrated an inversion pattern in situ, when we imaged the tumor ex vivo, there was significant fluorescence as compared with adjacent dura if the dura was removed along with the tumor (Fig. 2). Hence, even these 4 tumors demonstrated NIR fluorescence and thus ICG accumulation, albeit lower than in surrounding brain parenchyma. These 4 cases were not included in the sensitivity analysis of margins.

\section{Near-Infrared Contrast Dye to Detect Margins}

For each resection, the surgeon, at the time of surgery, examined the primary tumor specimen and coded it as either positive or negative with respect to white light. Fourteen of the 18 primary tumor resections showed significant
SBR fluorescence compared with surrounding normal brain. For the sensitivity and specificity analysis, we excluded the 4 cases with inversion. We reasoned that if the tumor showed poor fluorescence compared with surrounding brain, we could not trust the margins; thus, we did not use these tumor samples in the calculation of margin detection. Indeed, if the NIR imaging at the outset of surgery (before resection) demonstrated greater NIR signal in adjacent brain parenchyma, it would obviously make the surgeon wary of using these results for margin detection.

In the 14 cases in which the meningioma had a strong SBR as compared with adjacent brain parenchyma, an additional 32 margin samples were obtained (mean 2.3 margin samples per patient) for a total of 46 specimens. At 

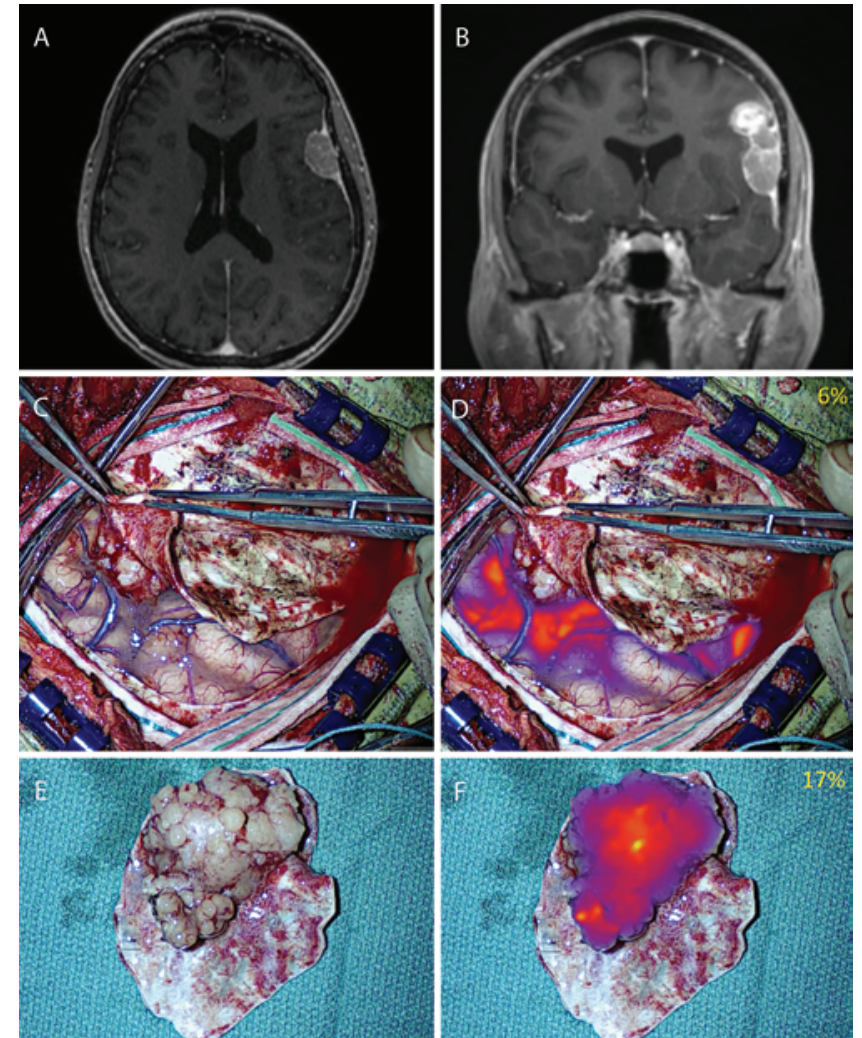

FIG. 2. Case 22. Convexity meningioma displaying inverted fluorescence signal. Axial (A) and coronal (B) contrast-enhanced T1-weighted MR images. In the visible-light view (C), the brain parenchyma can be identified and the tumor is just starting to be seen as the dura is peeled back. The NIR fluorescence superimposed and color-mapped onto the visible-light view (D) demonstrates an inverted pattern with more NIR signal in adjacent brain parenchyma than in tumor. The SBR of NIR tumor signal is 0.30 . When tumor was removed from the patient $(E)$, there was still accumulation of ${ }^{125} \mid \mathrm{R}$ dye within the meningioma as compared with the attached dura $(\mathbf{F})$. Ex vivo analysis confirms the presence of residual dye within tumor. Figure is available in color online only.

the time of biopsy, the surgeon coded the primary tumor specimens (14 specimens) plus the margin specimens (32 specimens) as positive or negative for tumor based on visible-light inspection and positive or negative for NIR signal (Table 3). Among the 46 specimens, 28 were positive for tumor (including 14 of the primary tumor specimens) and 18 were negative for tumor, according to the pathological diagnosis. The surgeon thought that 23 of the 46 specimens were positive for tumor under visible light. All 23 of those specimens came back positive for tumor, yielding a PPV of $100 \%$ for bright light (23/23). Using the NIR camera, the primary surgeon coded 38 of 46 specimens as positive. Of those 38 specimens, 27 were positive for tumor and 11 were negative for tumor (NIR false positives), yielding a PPV of $71 \%$ (27/38). Overall, the sensitivity of white light in predicting tumor was $82.1 \%$ (95\% CI 63.193.9) and its specificity was $100 \%$ (95\% CI $81.5-100)$. This gave a PPV of $100 \%$ (95\% CI 85.2-100), an NPV of 78.3\% (95\% CI 56.3-92.5), and an ROC area of 0.911. The NIR fluorescence camera system had a (better) sensitivity of 96.4\% (95\% CI 81.7-99.9), but its specificity decreased to $38.9 \%$ (95\% CI 17.3-64.3). The PPV was $71.1 \%$ (95\% CI
54.1-84.6), NPV was 87.5\% (95\% CI 47.3-99.7), and ROC area was 0.677 .

Because the pretest probability of biopsy is higher in atypical meningiomas since it is more likely that adjacent brain or dura will harbor tumor, we separately calculated the test characteristics for Grade I (11 cases) versus Grade II (3 cases) meningiomas. Again, we excluded the 4 patients with inversions. For the benign WHO Grade I meningiomas, 11 primary tumor samples were coded as positive under both bright light and NIR light. An additional 16 specimens were collected at the margins of the resection bed. Of the total 26 specimens of benign meningiomas, 14 were coded as positive under visible light and all 14 were pathologically confirmed to be WHO Grade I meningioma, yielding a PPV of 100\% (14/14). Twentyfour specimens were coded as positive under NIR light, and 17 were pathologically confirmed to be WHO Grade I meningioma, yielding a PPV of $71 \%$ (17/24). Under white light, the sensitivity of WHO Grade I meningioma detection was $82.4 \%$ (95\% CI 56.6-96.2), specificity was $100 \%$ (95\% CI 66.4-100), PPV was $100 \%$ (95\% CI 76.8-100), NPV was $75 \%$ (95\% CI 42.8-94.5), and ROC area was 0.912 (95\% CI 0.818-1). For the same subset of tumors under NIR light, sensitivity rose to $100 \%$ (95\% CI 80.5-100), but specificity fell to $22.2 \%$ (95\% CI 2.81-60). This gave a lower PPV of 70.8\% (95\% CI 48.9-87.4), NPV of $100 \%$ (95\% CI 15.8-100), and ROC area of 0.611.

The WHO Grade II atypical meningiomas are more likely to have adjacent residual tumor on a margin biopsy, and thus the pretest probability is higher in these cases. Twenty specimens from the 3 patients with atypical meningiomas were studied. Under bright light, 9 specimens were coded positive for tumor and all 9 specimens were pathologically proven to be WHO Grade II meningioma, yielding a PPV of $100 \%$ (9/9). Under NIR light, 10 specimens were coded as positive and all came back as tumor, yielding a PPV of $100 \%(10 / 10)$. Under bright light, the sensitivity of tumor resections for atypical meningiomas was $81.8 \%$ (95\% CI 48.2-97.7) and specificity was $100 \%$ (95\% CI 66.4-100). This gave a PPV of $100 \%$ (95\% CI 66.4-100), an NPV of $81.8 \%$ (95\% CI 48.2-97.7), and an ROC area of 0.909 (95\% CI 0.79-1). For the same subset of atypical tumors under NIR light, sensitivity rose to $90.9 \%$ (95\% CI 58.7-99.8), but specificity fell to $55.6 \%$ (95\% CI 21.2-86.3). This gave a lower PPV of 71.4\% (95\% CI 41.991.6), an NPV of 83.3 (95\% CI 35.9-99.6), and an ROC area of 0.732 .

In summary, for meningioma detection, bright light and the surgeon's impression yielded high sensitivity, specificity, PPV, and NPV. Near-infrared techniques utilizing second-window ICG administered 1 day before surgery resulted in higher sensitivity but at the expense of specificity and PPV. In higher-grade meningiomas (WHO Grade II), specificity improved but was still not as good as with bright light alone. Since the lack of specificity implies an excess of false positives, we sought to explain what might have contributed to this problem.

\section{Automatic Gain as an Explanation for Low Specificity}

In an effort to explore the cause of low specificity, we hypothesized that varying the NIR excitation interval was 
TABLE 2. Near-infrared signal to background ratio of primary tumor specimen

\begin{tabular}{|c|c|c|c|c|c|c|c|c|}
\hline Case No. & $\begin{array}{c}\text { Max Tumor } \\
\text { Diameter on } \\
\text { Preop MRI (mm) }\end{array}$ & $\begin{array}{l}\text { Ratio of T1 } \\
\text { Tumor Intensity/ } \\
\text { Normal Brain }\end{array}$ & $\begin{array}{l}\text { T2 FLAIR } \\
\text { Edema on } \\
\text { Preop MRI }\end{array}$ & $\begin{array}{c}\text { Ki-67 } \\
\text { (no. mitoses/hpf) }\end{array}$ & Endoscope & $\begin{array}{c}\text { Time From ICG } \\
\text { Injection to Camera } \\
\text { Visualization (hrs) }\end{array}$ & SBR & $\begin{array}{c}\text { Inverse } \\
\text { SBR }\end{array}$ \\
\hline \multicolumn{9}{|c|}{ Meningioma WHO Grade I } \\
\hline $1 \mathrm{~S}$ & 34.0 & 1.6 & 0 & 8 & & 22.2 & 6.05 & \\
\hline $13 S$ & 33.0 & 2.7 & 0 & 5 & & 22.1 & 5.23 & \\
\hline 9 & 23.9 & 2.9 & 1 & 3 & & 21.4 & 0.15 & Yes \\
\hline 21 & 85.4 & 1.5 & 1 & 2 & & 22.8 & 8.80 & \\
\hline 22 & 41.8 & 2.2 & 1 & 2 & & 27.0 & 0.30 & Yes \\
\hline 24 & 49.3 & 1.7 & 0 & 3 & & 21.8 & 5.35 & \\
\hline 29 & 39.8 & 1.8 & 0 & 3 & & 22.8 & 0.50 & Yes \\
\hline 31 & 19.8 & 1.9 & 0 & 1 & & 18.8 & 6.58 & \\
\hline 37 & 38.4 & 2.6 & 1 & 1 & & 18.0 & 7.79 & \\
\hline 40 & 45.2 & 2.0 & 1 & 1 & & 25.6 & 6.67 & \\
\hline 1 & 15.2 & 1.4 & 0 & 4 & Yes & 23.4 & 3.64 & \\
\hline 32 & 20.1 & 2.0 & 0 & 1 & Yes & 23.7 & 4.42 & \\
\hline 10 & 8.7 & 2.0 & 1 & 1 & Yes & 26.4 & 2.51 & \\
\hline 12 & 61.6 & 1.1 & 1 & & & 24.9 & 4.56 & \\
\hline 33 & 24.3 & 2.0 & 0 & 1 & Yes & 28.3 & 0.30 & Yes \\
\hline \multicolumn{9}{|c|}{ Meningioma WHO Grade II } \\
\hline $6 S$ & 70.0 & 1.9 & 0 & & & 19.1 & 5.08 & \\
\hline 17 & 17.4 & 1.5 & 0 & 20 & Yes & 24.0 & 4.98 & \\
\hline 3 & 22.3 & 2.7 & 1 & 5 & & 20.6 & 7.27 & \\
\hline
\end{tabular}

a possible source of variability and could explain the false positives. Our selected NIR camera system has an automatic exposure feature that averages the pixel intensity to normalize the background and then assigns it a neutral gray. As in other camera systems, if there is a bright window, the camera will close the aperture and lower the sensor gain to darken the image. Conversely, if there is a dark

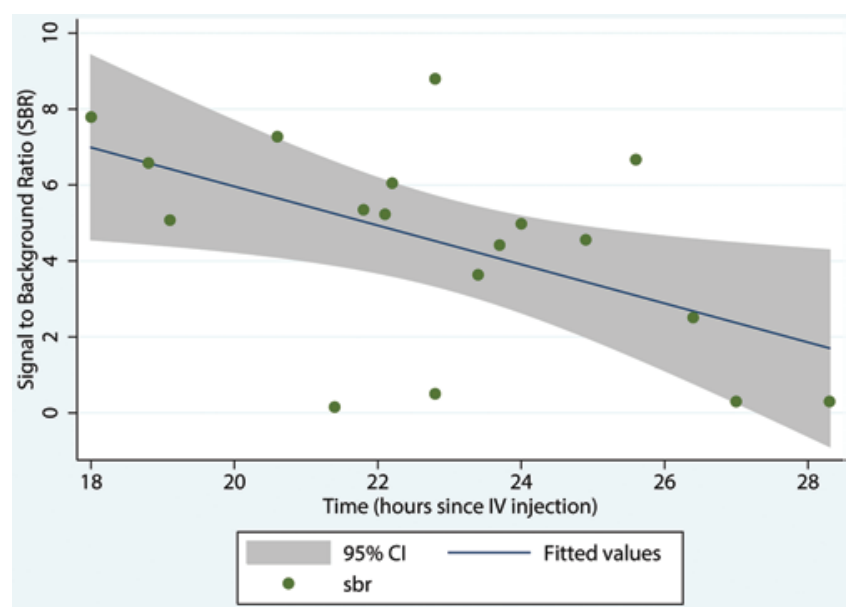

FIG. 3. Linear regression plot of SBR of NIR tumor signal versus time from intravenous (IV) infusion. The SBR decreases as the length of time progresses after infusion. The 4 cases with inversion are seen as the 4 cases with an $S B R<1 . p=0.022, R^{2}=0.2876$. Figure is available in color online only. room, the camera will open the aperture and increase the sensor gain to brighten the image. We discovered that this auto-correction might contribute to the false-positive rate in the NIR spectrum. If there was minimal NIR signal, such as after the resection of primary tumor (which has a strong NIR signal), the camera would increase the sensor exposure time to "find" or "overexpose" areas of NIR (Fig. 4). This could result in false positives and thus lower specificity.

In a case of atypical WHO Grade II meningioma (Case 6S; Tables 1 and 2), the primary tumor visualized by the VisionSense iridium camera demonstrated a gain percent of $20 \%$ and an SBR of 6.08. The primary tumor was resected, and an additional 14 margins were biopsied. When visualized by the camera with the automatic exposure time function, some samples were harvested because the signal was very strong, but the gain percentage was not specifically considered during the biopsy procedure. On post hoc analysis, we found that the gain percent for the 7 pathologically positive specimens was a mean $21.1 \% \pm$ $2.5 \%$ (range 9\%-29\%), whereas the gain percent for the 8 pathologically negative tumors was $68.5 \% \pm 10.4 \%$ (range $35 \%-100 \%)$. Indeed, when the gain percent was allowed to drift above $30 \%$ (50\% greater than the gain at which the original tumor was imaged, in this case $20 \%$ ), the falsepositive detection by NIR increased significantly. There were no false positives when the gain percent was kept below 30\%, whereas all 4 false positives occurred when the gain percent was allowed to drift above $30 \%$. An example of this can be seen in Fig. 4, which features Case 13S. 
TABLE 3. Test characteristics of white light versus NIR in 14 patients

Test Characteristics of White Light in 46 Specimens

\begin{tabular}{|c|c|c|c|c|c|c|c|c|}
\hline & & \multicolumn{2}{|c|}{$\begin{array}{l}\text { Positive for Tumor Under White } \\
\text { Light, According to Surgeon }\end{array}$} & \multirow{2}{*}{$\begin{array}{r}\text { Sensitivity } \\
(95 \% \mathrm{Cl})\end{array}$} & \multirow{2}{*}{$\begin{array}{c}\text { Specificity } \\
(95 \% \mathrm{Cl})\end{array}$} & \multirow{2}{*}{$\begin{array}{c}\text { PPV } \\
(95 \% \mathrm{Cl})\end{array}$} & \multirow{2}{*}{$\begin{array}{c}\text { NPV } \\
(95 \% \mathrm{Cl})\end{array}$} & \multirow{2}{*}{$\begin{array}{c}\text { ROC Area } \\
(95 \% \mathrm{Cl})\end{array}$} \\
\hline & & Yes & No & & & & & \\
\hline \multirow{2}{*}{$\begin{array}{l}\text { Tumor pathology according to } \\
\text { ultimate pathological diagnosis }\end{array}$} & Yes & 23 & 5 & \multirow{2}{*}{$\begin{array}{c}82.1 \% \\
(63.1-93.9)\end{array}$} & \multirow{2}{*}{$\begin{array}{c}100 \% \\
(81.5-100)\end{array}$} & \multirow{2}{*}{$\begin{array}{c}100 \% \\
(85.2-100)\end{array}$} & \multirow{2}{*}{$\begin{array}{c}78.3 \% \\
(56.3-92.5)\end{array}$} & \multirow{2}{*}{$\begin{array}{c}0.911 \\
(0.838-0.983)\end{array}$} \\
\hline & No & 0 & 18 & & & & & \\
\hline
\end{tabular}

Test Characteristics of NIR in 46 Specimens

\begin{tabular}{|c|c|c|c|c|c|c|c|c|}
\hline & & \multicolumn{2}{|c|}{$\begin{array}{l}\text { Positive for Tumor Under NIR } \\
\text { Camera, According to Surgeon }\end{array}$} & \multirow{2}{*}{$\begin{array}{r}\text { Sensitivity } \\
(95 \% \mathrm{Cl})\end{array}$} & \multirow{2}{*}{$\begin{array}{c}\text { Specificity } \\
(95 \% \mathrm{Cl})\end{array}$} & \multirow{2}{*}{$\begin{array}{c}\text { PPV } \\
(95 \% \mathrm{Cl})\end{array}$} & \multirow{2}{*}{$\begin{array}{c}\text { NPV } \\
(95 \% \mathrm{Cl})\end{array}$} & \multirow{2}{*}{$\begin{array}{c}\text { ROC Area } \\
(95 \% \mathrm{Cl})\end{array}$} \\
\hline & & Yes & No & & & & & \\
\hline \multirow{2}{*}{$\begin{array}{l}\text { Tumor pathology according to } \\
\text { ultimate pathological diagnosis }\end{array}$} & Yes & 27 & 1 & \multirow{2}{*}{$\begin{array}{c}96.4 \% \\
(81.7-99.9)\end{array}$} & \multirow{2}{*}{$\begin{array}{c}38.9 \% \\
(17.3-64.3)\end{array}$} & \multirow{2}{*}{$\begin{array}{c}71.1 \% \\
(54.1-84.6)\end{array}$} & \multirow{2}{*}{$\begin{array}{c}87.5 \% \\
(47.3-99.7)\end{array}$} & \multirow{2}{*}{$\begin{array}{c}0.677 \\
(0.556-0.798)\end{array}$} \\
\hline & No & 11 & 7 & & & & & \\
\hline
\end{tabular}

\section{Discussion}

Fluorescence-guided tumor resection provides a novel approach for visualizing tumor above and beyond visiblelight inspection. Most current reports on meningioma surgery focus on the use of 5-ALA, a protoporphyrin prodrug that is metabolized by ferrochelatase. Investigators have described the strong, homogeneous visible red fluorescence that is observed in meningioma surgery. ${ }^{39}$ In addition, the diagnostic accuracy of 5-ALA is approximately $90 \%$ when comparing normal dura to actual tumor. Bone invasion can be identified as well. ${ }^{10}$ Thus, 5-ALA is a promising visible light fluorophore for meningioma surgery ${ }^{8}$ however, it has a significant limitation arising from its excitation and activation in the visible light spectrum $(450-650 \mathrm{~nm})$, and it is not FDA-approved for use in the United States.

Near-infrared fluorescence imaging has several advantages over visible light fluorescence imaging. First, tissue penetration is related to wavelength, and the longer the wavelength the greater the depth of penetration. Hence, NIR fluorophores (700-900 nm) provide superior detection of residual fluorescence. ${ }^{37}$ The tissue penetration of ICG is approximately $8-10 \mathrm{~mm}$ in brain tissue, whereas the depth of penetration of 5-ALA is approximately 3-5 $\mathrm{mm}$. Second, NIR imaging does not interfere with the visible light spectrum; thus, there is no autofluorescence from collagens or heme products during neurosurgical procedures. This permits real-time dual imaging using a standard visible-light view with the full spectrum of red to blue colors while capturing NIR fluorophore emission. These images can be spatially coregistered, providing anatomical contrast and precision.

To date, ICG has been used primarily as a vascular contrast agent for angiography. ${ }^{33}$ It is a water-soluble molecule with a hydrodynamic diameter of $1.2 \mathrm{~nm}$ and excitation and emission wavelengths in serum at 778 and $830 \mathrm{~nm}$, respectively. It is rapidly bound to plasma proteins and rapidly metabolized by the liver and thus has a half-life of approximately 5 minutes ("first-window ICG"). It has been in clinical use since the 1950s for ophthalmic angiography, determining cardiac output and hepatic function measurements, and is FDA approved. However, it has only recently shown real value in tumor identification for surgical oncology. ${ }^{17,21,25,28,34}$ The second-window ICG technique, in which a high dose of ICG is delivered approximately 24 hours before visualization, can be used to deliver ICG to tumors via a passive targeting mechanism called the EPR effect. $^{12,26}$

The EPR effect was initially described in 1986 by Matsumura and Maeda. ${ }^{26}$ For the past 30 years, this phenomenon has been accepted in the field of drug delivery as an important means of nanoparticle accumulation within tumors. ${ }^{3,23}$ When solid tumor reaches a certain critical size, it outgrows the normal surrounding vasculature's ability to supply critical nutrients; therefore, tumor cells will stimulate a complex process of tumor angiogenesis, including the production of vascular permeability factors to deliver oxygen and nutrients. However, these tumor-related vessels are characterized by defective endothelial cells with wide fenestrations, which increase vessel permeability. These fenestrations range from 200 to $2000 \mathrm{~nm}$, depending on tumor type and location. ${ }^{16}$ Nanoparticles reach the abnormal vasculature within the tumor, meet with little resistance, and thus permeate the tumor. In normal tissues, even those in the brain, lymphatic drainage proceeds at a consistent rate of $0.1-2 \mu \mathrm{m} / \mathrm{second}^{3}$ and provides a continual source of fluid and molecular exchange within the interstitial extracellular fluid matrix. In tumors, this lymphatic drainage does not function properly, and thus nanoparticles can be retained..$^{23}$ As a consequence, small molecules such as ICG can readily permeate the cancer tissues and then accumulate (retained) without proper clearance. This EPR effect leads to an accumulation of small nanoparticle-sized molecules in diseased tissues. Most recently, investigators have exploited the EPR effect for intraoperative visualization.

The senior author (S.S.) has investigated the EPR technique for tumor visualization in rodent, canine, and human studies. ${ }^{18,21,34}$ An important rodent study was recently published by Jiang et al. ${ }^{18}$ These investigators from the University of Pennsylvania used a murine flank lung tumor model to contrast videoangiography with NIR imaging of tumor accumulation of ICG injected via the tail vein at doses ranging from 0 to $10 \mathrm{mg} / \mathrm{kg}$. The NIR signal in flank tumor was contrasted with that in peripheral blood vasculature by using an NIR camera system. At standard videoangiography doses of $0.7 \mathrm{mg} / \mathrm{kg}$, the tumor could not 


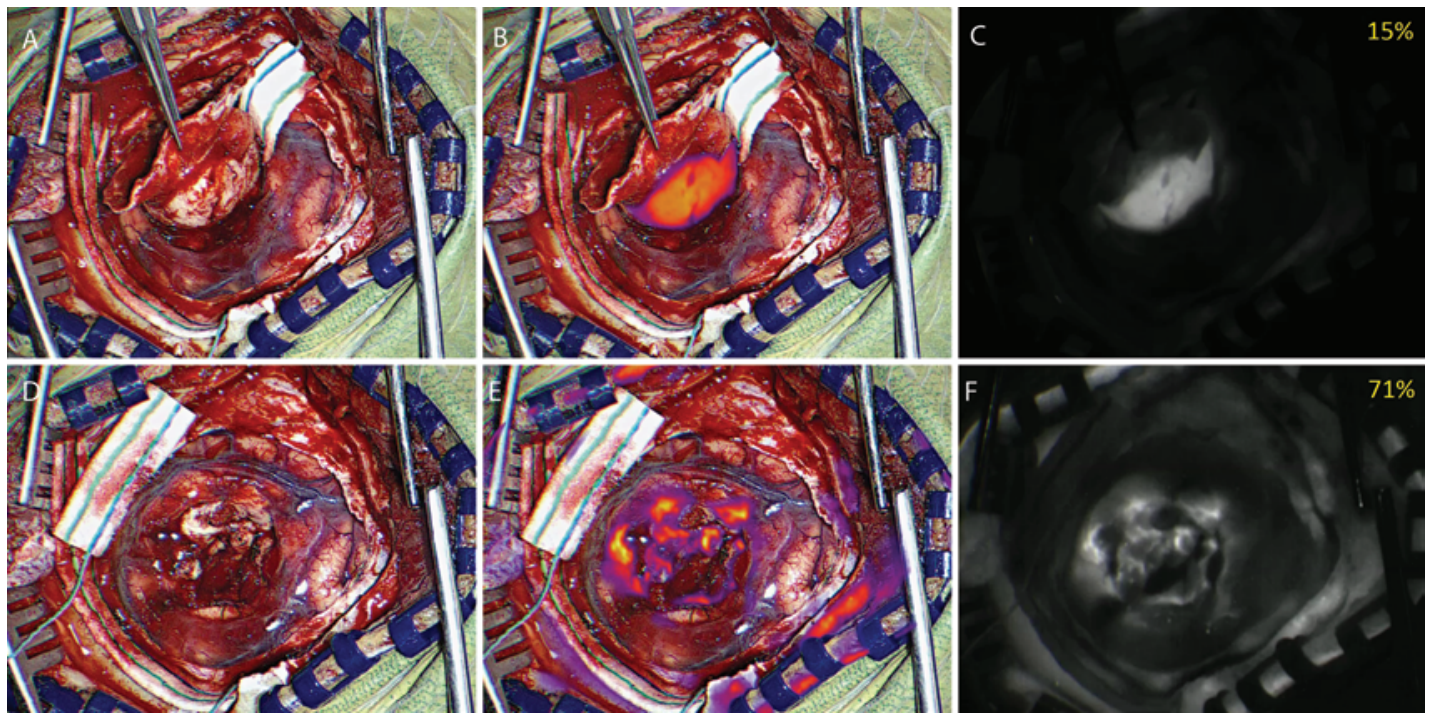

FIG. 4. Case 13S. Automatic gain function may contribute to false positives. The visible-light image (A), NIR fluorescence superimposed and color-mapped onto the visible-light view (B), and NIR image (C) reveal the main specimen displaying strong NIR signal with a gain percentage at $15 \%$. The visible-light image (D), NIR fluorescence superimposed and color-mapped onto the visiblelight view (E), and NIR image (F) obtained after resection of the main tumor, showing a gain percentage increase to $71 \%$. Notice the presence of a significant increase in background NIR signal in the skin edges partially obscured by Raney clips, bone, and so forth. Biopsy did not confirm the presence of meningioma. Figure is available in color online only.

be visualized at all. Upon administration of higher doses of $2.5-10 \mathrm{mg} / \mathrm{kg}$, the tumor could be visualized with NIR imaging. Surprisingly, the tumor retained the strong NIR signal for a period that extended much beyond the half-life of ICG in the blood. The NIR fluorescence in the flank tumor reached a peak at 30 minutes, remained at a relatively high plateau for up to 24 hours, and then gradually decreased over time. In contrast, NIR fluorescence in the vasculature peaked immediately upon administration of the dye and gradually decayed over the 72-hour period of the study. The authors plotted the ratio of NIR fluorescence of tumor to NIR fluorescence of vasculature and concluded that the optimal tumor to vascular NIR fluorescence was observed at 24 hours. In addition, the investigators concluded that the NIR signal was $15 \%$ higher at $10 \mathrm{mg}$ / $\mathrm{kg}$ as compared with $5 \mathrm{mg} / \mathrm{kg}$, but this was not subjectively noticeable; thus, their human trials in lung cancer started at a dose of $5 \mathrm{mg} / \mathrm{kg}$ of ICG and visualization delayed for 24 hours. These studies in lung cancer have yielded favorable results. $18,21,34$

Given the favorable results in lung cancer, we focused our efforts on the most common primary brain tumor in humans-meningioma. Our primary purpose in the current study was to see if second-window ICG could be safely administered 24 hours prior to surgery and whether NIR fluorescence could be visualized within the meningioma tissue at the time of surgery. We administered ICG at a $5-\mathrm{mg} / \mathrm{kg}$ dose, which is below the lethal dose, $50 \%$ for human administration but more than 10 times the usual dose used for angiography (25-mg aliquots, which is approximately $0.35 \mathrm{mg} / \mathrm{kg}$ for a $70-\mathrm{kg}$ human). In addition, although the half-life of ICG is less than 10 minutes, sufficient dye remained within the tumor tissue for visualization approximately 24 hours later, known as the "second window."
All of the tumors demonstrated accumulation of the NIR second-window ICG at the time of surgery, but only 14 of the 18 patients demonstrated appropriate SBR compared with normal brain upon initial exposure. Upon careful review, we recognized that the inversion pattern seen in the other 4 patients could be related to timing; it is possible that the NIR dye diffused out of the tumor into adjacent brain parenchyma because of the prolonged time interval from injection to imaging. Indeed, all inversions were observed in patients whose imaging had been performed more than 21 hours after dye injection (Fig. 3). We acknowledge that drug accumulation within tumor parenchyma is incompletely understood and quite heterogeneous. As stated in a recent review on the EPR effect, Bertrand et al. state that at least 3 interrelated phenomena influence drug accumulation: extravasation of drug, diffusion of drug through tumor, and interaction of drug with both intracellular and extracellular targets. ${ }^{3}$ The third issue is challenging to understand, especially since we recognize that all meningiomas are not the same molecularly 5,6 and thus that the interactions of ICG with the intracellular and extracellular targets can vary, explaining our heterogeneous findings.

Another major finding of this study is based on the autoexposure of the camera system and the rate of falsepositive margins. When the camera is moved into position above the tumor, the camera adjusts in an automatic fashion to find an NIR signal. This adjustment is known as the gain percent, ranging from $0 \%$ to $100 \%$. When a strong NIR signal is found in the field, the gain percent is low as the camera does not need to fully open its aperture and overexpose to find a signal. The weaker the NIR signal, the higher the gain percent until it reaches its maximum capacity at $100 \%$. We observed that when the main tumor is being removed, the gain percent of the camera system 
is very low as there is a strong concentration of ICG in the tumor. After the main tumor is resected, the gain percent then climbs to a much higher value as the camera is searching for an NIR signal in the margins. We believe the low specificity of the margin biopsies is attributable to the fact that the camera automatically adjusted to a much higher gain percent and thus was falsely identifying positive margins, which were in fact much less NIR positive than the main tumor. Given this trend, we recently adjusted our practice of taking margins by not allowing the sensor to adjust the gain in an automatic fashion. We now manually fix the gain percent when the main tumor is being resected and thus prevent it from automatically adjusting to a higher setting to detect a weaker signal.

Second-window ICG is not receptor specific. The NIR dye binds to plasma albumin within the circulation, and we believe that at the high concentration administered, second-window ICG remains extracellular, within the tumor matrix, and thus does not bind to a specific receptor. ${ }^{22,32}$ This, in turn, may cause the low specificity of second-window ICG for tumor. Thus, the second-window ICG technique has several limitations. We suspect that high false positives were seen because of the use of the automatic gain feature of the camera system. Moreover, the timing of imaging must be optimized, and we recommend imaging second-window ICG less than 21 hours after intravenous infusion.

We acknowledge that there are limitations to this study. It is a pilot study; therefore, the study sample was small (18 cases). The sensitivity and specificity tests are largely determined by sample size, and thus accurate test calculation was limited. In addition, the SBR is an arbitrary number and may not be a true reflection of tissue fluorescence. Lastly, the study was performed by 1 surgeon and may be subject to selection bias and other unforeseen limitations.

\section{Conclusions}

In summary, although many NIR fluorescent agents are currently available, second-window ICG is the only fluorescent drug currently approved for intracranial use in the United States by the FDA. ${ }^{28}$ With its relatively low cost, widespread availability, and minimal toxicity, ICG serves as a reasonable and practical NIR contrast agent choice. ${ }^{11,17,19}$ We demonstrated that meningiomas, both WHO Grade I and II, fluoresce in real time during surgery by using an NIR-capable camera. The sensitivity of second-window ICG for tumor appears to be better than the surgeon's impression using white light alone, but the current technique has lower specificity for tumor than white light alone. We suggest a technique (fixing the exposure time) that may provide better specificity in the future. Nevertheless, we believe this is the first study to demonstrate that second-window ICG can accumulate in meningioma and be used as a means of identifying meningioma tissue in real time during surgery for meningioma resection in humans. Further work must be done to optimize this technique, and we continue to seek methods to refine our results and we look forward to the contributions of neurosurgeons throughout the world.

\section{Acknowledgments}

This work was partially supported by the National Institutes of Health Grant No. R01 CA193556 (S.S.) and the Institute for Translational Medicine and Therapeutics of the Perelman School of Medicine at the University of Pennsylvania (J.Y.K.L.). In addition, research reported in this publication was supported by the National Center for Advancing Translational Sciences of the National Institutes of Health under Award No. UL1TR000003 (J.Y.K.L.). The content is solely the responsibility of the authors and does not necessarily represent the official views of the NIH.

\section{References}

1. Alacam B, Yazici B, Intes X, Chance B: Analysis of ICG pharmacokinetics in cancerous tumors using NIR optical methods. Conf Proc IEEE Eng Med Biol Soc 1:62-65, 2005

2. Barker FG II, Curry WT Jr, Carter BS: Surgery for primary supratentorial brain tumors in the United States, 1988 to 2000: the effect of provider caseload and centralization of care. Neuro Oncol 7:49-63, 2005

3. Bertrand N, Wu J, Xu X, Kamaly N, Farokhzad OC: Cancer nanotechnology: the impact of passive and active targeting in the era of modern cancer biology. Adv Drug Deliv Rev 66:2-25, 2014

4. Borovich B, Doron Y: Recurrence of intracranial meningiomas: the role played by regional multicentricity. J Neurosurg 64:58-63, 1986

5. Brastianos PK, Horowitz PM, Santagata S, Jones RT, McKenna A, Getz G, et al: Genomic sequencing of meningiomas identifies oncogenic SMO and AKT1 mutations. Nat Genet 45:285-289, 2013

6. Clark VE, Erson-Omay EZ, Serin A, Yin J, Cotney J, Ozduman K, et al: Genomic analysis of non-NF2 meningiomas reveals mutations in TRAF7, KLF4, AKT1, and SMO. Science 339:1077-1080, 2013

7. Claus EB, Bondy ML, Schildkraut JM, Wiemels JL, Wrensch M, Black PM: Epidemiology of intracranial meningioma. Neurosurgery 57:1088-1095, 2005

8. Coluccia D, Fandino J, Fujioka M, Cordovi S, Muroi C, Landolt H: Intraoperative 5-aminolevulinic-acid-induced fluorescence in meningiomas. Acta Neurochir (Wien) 152:1711-1719, 2010

9. Della Puppa A, Rustemi O, Gioffrè G, Rolma G, Grandis M, Munari M, et al: Application of indocyanine green video angiography in parasagittal meningioma surgery. Neurosurg Focus 36(2):E13, 2014

10. Della Puppa A, Rustemi O, Gioffrè G, Troncon I, Lombardi G, Rolma G, et al: Predictive value of intraoperative 5-aminolevulinic acid-induced fluorescence for detecting bone invasion in meningioma surgery. J Neurosurg 120:840-845, 2014

11. Ferroli P, Acerbi F, Albanese E, Tringali G, Broggi M, Franzini A, et al: Application of intraoperative indocyanine green angiography for CNS tumors: results on the first 100 cases. Acta Neurochir Suppl 109:251-257, 2011

12. Greish K: Enhanced permeability and retention (EPR) effect for anticancer nanomedicine drug targeting. Methods Mol Biol 624:25-37, 2010

13. Haglund MM, Berger MS, Hochman DW: Enhanced optical imaging of human gliomas and tumor margins. Neurosurgery 38:308-317, 1996

14. Haglund MM, Hochman DW, Spence AM, Berger MS, Kaye $\mathrm{AH}$, Kelly PJ, et al: Enhanced optical imaging of rat gliomas and tumor margins. Neurosurgery 35:930-941, 1994

15. Hansen DA, Spence AM, Carski T, Berger MS: Indocyanine green (ICG) staining and demarcation of tumor margins in a rat glioma model. Surg Neurol 40:451-456, 1993

16. Hobbs SK, Monsky WL, Yuan F, Roberts WG, Griffith L, 
Torchilin VP, et al: Regulation of transport pathways in tumor vessels: role of tumor type and microenvironment. Proc Natl Acad Sci U S A 95:4607-4612, 1998

17. Holt D, Okusanya O, Judy R, Venegas O, Jiang J, DeJesus E, et al: Intraoperative near-infrared imaging can distinguish cancer from normal tissue but not inflammation. PLoS One 9:e103342, 2014

18. Jiang JX, Keating JJ, Jesus EM, Judy RP, Madajewski B, Venegas O, et al: Optimization of the enhanced permeability and retention effect for near-infrared imaging of solid tumors with indocyanine green. Am J Nucl Med Mol Imaging 5:390-400, 2015

19. Kim EH, Cho JM, Chang JH, Kim SH, Lee KS: Application of intraoperative indocyanine green videoangiography to brain tumor surgery. Acta Neurochir (Wien) 153:14871495,2011

20. Kubben PL, ter Meulen KJ, Schijns OE, ter Laak-Poort MP, van Overbeeke JJ, van Santbrink H: Intraoperative MRIguided resection of glioblastoma multiforme: a systematic review. Lancet Oncol 12:1062-1070, 2011

21. Madajewski B, Judy BF, Mouchli A, Kapoor V, Holt D, Wang $\mathrm{MD}$, et al: Intraoperative near-infrared imaging of surgical wounds after tumor resections can detect residual disease. Clin Cancer Res 18:5741-5751, 2012

22. Maeda H, Greish K, Fang J: The EPR effect and polymeric drugs: a paradigm shift for cancer chemotherapy in the 21st century. Adv Polym Sci 193:103-121, 2006

23. Maeda H, Tsukigawa K, Fang J: A retrospective 30 years after discovery of the enhanced permeability and retention effect of solid tumors: next-generation chemotherapeutics and photodynamic therapy-problems, solutions, and prospects. Microcirculation 23:173-182, 2016

24. Maeda H, Wu J, Sawa T, Matsumura Y, Hori K: Tumor vascular permeability and the EPR effect in macromolecular therapeutics: a review. J Control Release 65:271-284, 2000

25. Marshall MV, Rasmussen JC, Tan IC, Aldrich MB, Adams $\mathrm{KE}$, Wang X, et al: Near-infrared fluorescence imaging in humans with indocyanine green: a review and update. Open Surg Oncol J 2:12-25, 2010

26. Matsumura Y, Maeda H: A new concept for macromolecular therapeutics in cancer chemotherapy: mechanism of tumoritropic accumulation of proteins and the antitumor agent smancs. Cancer Res 46:6387-6392, 1986

27. Mirimanoff RO, Dosoretz DE, Linggood RM, Ojemann RG, Martuza RL: Meningioma: analysis of recurrence and progression following neurosurgical resection. J Neurosurg 62:18-24, 1985

28. Okusanya OT, Madajewski B, Segal E, Judy BF, Venegas OG, Judy RP, et al: Small portable interchangeable imager of fluorescence for fluorescence guided surgery and research. Technol Cancer Res Treat 14:213-220, 2015

29. Olar A, Wani KM, Sulman EP, Mansouri A, Zadeh G, Wilson $\mathrm{CD}$, et al: Mitotic Index is an independent predictor of recurrence-free survival in meningioma. Brain Pathol 25:266-275, 2015

30. Orringer DA, Golby A, Jolesz F: Neuronavigation in the surgical management of brain tumors: current and future trends. Expert Rev Med Devices 9:491-500, 2012

31. Pollock BE, Stafford SL, Utter A, Giannini C, Schreiner SA: Stereotactic radiosurgery provides equivalent tumor control to Simpson Grade 1 resection for patients with small- to medium-size meningiomas. Int J Radiat Oncol Biol Phys 55:1000-1005, 2003
32. Predina JD, Judy B, Fridlender ZG, Aliperti LA, Madajewski B, Kapoor V, et al: A positive-margin resection model recreates the postsurgical tumor microenvironment and is a reliable model for adjuvant therapy evaluation. Cancer Biol Ther 13:745-755, 2012

33. Scerrati A, Della Pepa GM, Conforti G, Sabatino G, Puca A, Albanese A, et al: Indocyanine green video-angiography in neurosurgery: a glance beyond vascular applications. Clin Neurol Neurosurg 124:106-113, 2014

34. Singhal S, Nie S, Wang MD: Nanotechnology applications in surgical oncology. Annu Rev Med 61:359-373, 2010

35. Stummer W, Novotny A, Stepp H, Goetz C, Bise K, Reulen HJ: Fluorescence-guided resection of glioblastoma multiforme by using 5-aminolevulinic acid-induced porphyrins: a prospective study in 52 consecutive patients. J Neurosurg 93:1003-1013, 2000

36. Stummer W, Pichlmeier U, Meinel T, Wiestler OD, Zanella F, Reulen HJ: Fluorescence-guided surgery with 5-aminolevulinic acid for resection of malignant glioma: a randomised controlled multicentre phase III trial. Lancet Oncol 7:392401, 2006

37. Vahrmeijer AL, Hutteman M, van der Vorst JR, van de Velde $\mathrm{CJH}$, Frangioni JV: Image-guided cancer surgery using nearinfrared fluorescence. Nat Rev Clin Oncol 10:507-518, 2013

38. Valdes PA, Bekelis K, Harris BT, Wilson BC, Leblond F, Kim A, et al: 5-Aminolevulinic acid-induced protoporphyrin IX fluorescence in meningioma: qualitative and quantitative measurements in vivo. Neurosurgery 10 (Suppl 1):74-83, 2014

39. Valdés PA, Leblond F, Kim A, Harris BT, Wilson BC, Fan $X$, et al: Quantitative fluorescence in intracranial tumor: implications for ALA-induced PpIX as an intraoperative biomarker. J Neurosurg 115:11-17, 2011

40. Zhao S, Wu J, Wang C, Liu H, Dong X, Shi C, et al: Intraoperative fluorescence-guided resection of high-grade malignant gliomas using 5-aminolevulinic acid-induced porphyrins: a systematic review and meta-analysis of prospective studies. PLoS One 8:e63682, 2013

\section{Disclosures}

Dr. Lee owns stock options in VisionSense. Dr. Singhal holds patent rights over technologies presented in this paper.

\section{Author Contributions}

Conception and design: Lee, Pierce, Thawani, Nie, Singhal. Acquisition of data: Lee, Pierce, Zeh, Martinez-Lage. Analysis and interpretation of data: all authors. Drafting the article: all authors. Critically revising the article: all authors. Reviewed submitted version of manuscript: all authors. Approved the final version of the manuscript on behalf of all authors: Lee. Statistical analysis: Lee, Pierce, Zeh, Nie, Martinez-Lage, Singhal. Administrative/technical/material support: Lee, Pierce, Zeh, Singhal. Study supervision: Lee, Thawani, Singhal.

\section{Correspondence}

John Y. K. Lee, Department of Neurosurgery, University of Pennsylvania, 235 South Eighth St., Philadelphia, PA 19106. email: leejohn@uphs.upenn.edu. 